\title{
Understanding Flow through Catalytic Converters
}

\author{
Hesham A. Ibrahim ${ }^{1}$, Sherif Abdou ${ }^{2}$, and Wael H. Ahmed ${ }^{1}$ \\ ${ }^{1}$ University of Guelph \\ 50 Stone Rd, Guelph, Ontario, Canada \\ hesham@mail.uoguelph.ca; ahmedw@uoguelph.ca \\ ${ }^{2}$ Vida Fresh Air Corp. \\ 2000 Argentia Rd, Mississauga, Ontario, Canada \\ sherif.a@vidafreshair.com
}

\begin{abstract}
In this experiment the fluid flow behaviour through an automotive catalytic converter has been studied empirically and computationally. A CFD model utilizing the $\mathrm{k}-\omega$ turbulence model and porous media approach was developed to simulate the flow through the monolithic ceramic substrate. The flow properties for the ceramic monolith were obtained using a $3 \mathrm{D}$ single channel approach. CFD analysis was validated by flow experiments with non-reacting flow using high temperature air. The local velocity and temperature profiles for inlet Reynolds Number of 43,000 were measured using hot-wire and high sensitivity thermocouple sensors. The CFD results were found to predict the experimental measurements with an average root mean square error of $\pm 8 \%$. The present analysis is considered to be a key step towards understanding the flow behaviour through catalytic converters that can help in achieving better design of the exhaust system.
\end{abstract}

Keywords: Flow Distribution; Catalytic Converter; Thermalhydraulic Performance

\section{Introduction}

The need for better automotive technologies in order to improve fuel economy, while meeting new stringent global vehicle emission standards, continues to grow with the increasing demand for environmental protection. The primary emissions of carbon monoxide, unburnt hydrocarbons and nitrogen oxides from the engine represent a negative impact on both the environment and public health [1,2]. Early catalytic converter designs reduced emissions, but at the cost of increased engine back pressure and fuel consumption[3].

Current catalytic converter designs attempt to overcome these drawbacks using a cordierite ceramic monolith formed into a honeycomb-like structure with straight channels. This design offers lower back pressure and higher conversion efficiency of pollutants due to better flow distribution[4]. Further improvements of ceramic monolith design necessitates intensive computational and experimental work to optimize the complex interaction between chemistry, thermalhydraulic and materials effects on the performance of the converter. Computational fluid dynamics (CFD) simulations and mathematical modeling offer an opportunity to minimize cost and time consumption[5].

Flow behaviour inside a catalytic converter has been studied previously, focusing on the effects of exhaust manifold design (inlet/outlet configuration) and monolith variability (wall thickness and cell density) [6,7]. Flow uniformity was increased by utilizing higher cell density monoliths with smaller hydraulic diameter and by splitting the monolith into two parts separated by a gap [8-11]. Lower flow uniformity was observed in 3D steady state and transient numerical simulations in systems with higher monolith-to-inlet diameter ratios [12,13]. In particular, the design of the inlet diffuser was found to play an important role in the converter design $[14,15]$.

In the present study, a CFD model is developed taking into consideration the thermal effects on the performance of the catalytic converter and is validated against both local temperature and velocity experimental measurements. A controlled hot air experimental setup is used to investigate the flow distribution under steady state conditions at the desired temperature and flow rate. 


\section{Experimental Setup}

The principal objective of the experiment is to measure temperature and velocity profiles downstream of the ceramic monolith of the catalytic converter. The experimental setup is designed to mimic a typical automotive exhaust system using steady hot air flow (see Figure 1a). The test section consists of a straight vertical inlet pipe with an inlet diameter of $5 \mathrm{~cm}$ and length of $53 \mathrm{~cm}$, and utilizes a $7.6 \mathrm{~cm}$ diameter and $7.6 \mathrm{~cm}$ long monolith container. The exit pipe is $18 \mathrm{~cm}$ long and 5 $\mathrm{cm}$ in diameter. Both inlet and exit diffusers have a cone angle of $45^{\circ}$ and are $2.5 \mathrm{~cm}$ long. Measurements were recorded for a mass flow rate of $0.05 \mathrm{~kg} / \mathrm{s}$ and an inlet air temperature of $177^{\circ} \mathrm{C}$ under steady state conditions $(\operatorname{Re} \sim 43,000$ based on inlet pipe diameter). The catalytic converter assembly consists of a monolith with a cell density of 400 Cells Per Square Inch (CPSI) and a wall thickness of 6.5 mil $\left(6.5 \times 10^{-3} \mathrm{inch}\right)$. The monolith is insulated using an insulation mat wrapped around the monolith with a thickness of $3 \mathrm{~mm}$. The monolith and insulation are inserted in a stainless steel cylindrical canto form the catalytic converter assembly as shown in Figure 1b.

A controlled air flow rate ranging from 0 to $4500 \mathrm{~L} / \mathrm{min}$ is supplied by a high pressure air blower driven by a variable speed motor and the average flow rate is measured using an air rotameter. The air is heated by an $11 \mathrm{~kW}$ electric heater (temperature range $50-650{ }^{\circ} \mathrm{C}$ ) before passing through the vertical test section. The temperature is monitored using three bulk thermocouples with an accuracy of $\pm 0.5^{\circ} \mathrm{C}$ distributed along the inlet and outlet test sections. Pressure drop measurements across the test section are taken using a differential pressure transducer connected upstream and downstream of the catalytic converter with an accuracy of $\pm 17 \mathrm{kPa}$. The velocity measurements are taken using a high temperature hotwire probe (Dantec Dynamics Model 55A75) with an accuracy of $\pm 5 \%$ and mounted on a traverse mechanism with a minimum traversing step of $0.0025 \mathrm{~mm}$. Temperature profiles are measured using a T-type thermocouple with an accuracy of $\pm 0.5^{\circ} \mathrm{C}$ mounted on a separate traverse. Both radial velocity and temperature measurements were taken every 3 -mm along the catalytic converter radius. The plane at which the temperature and velocity profiles are measure is $0.5 \mathrm{~cm}$ downstream of the monolith. The temperature signal is sampled for 10 second with a sampling frequency of $100 \mathrm{~Hz}$. The velocity signal is sampled for 40 seconds with a sampling frequency of $3 \mathrm{kHz}$.

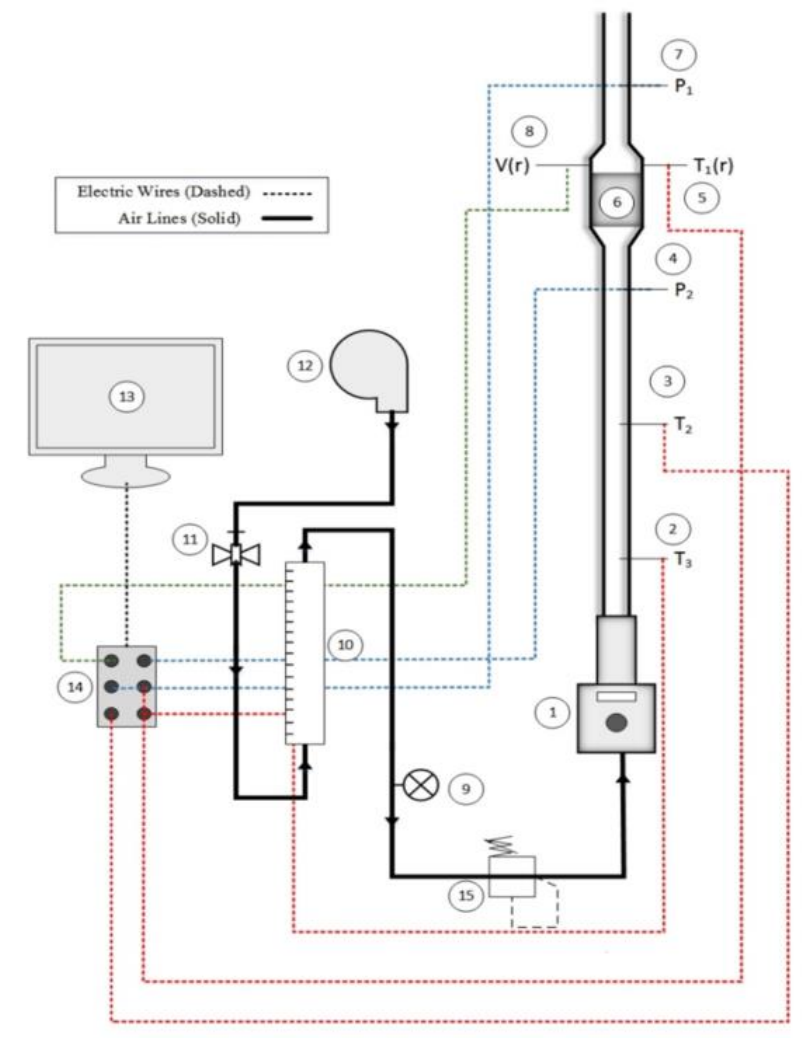

(a)
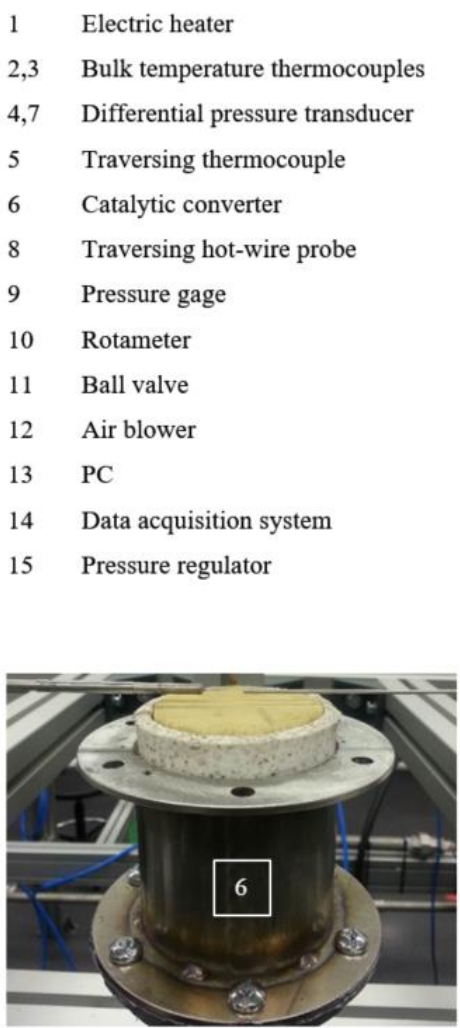

(b)

Fig. 1: (a) Schematic of experimental setup and (b) actual image of catalytic converter housing. 


\section{Mathematical Modelling}

Modelling of the flow through the catalytic converter is carried out using the commercial Ansys Fluent CFD package. The monolith is treated as a porous zone where inertial and viscous terms are defined by Darcy's law [14] and are obtained by conducting discrete channel simulations for a single monolith flow channel at the given range of flow conditions. The flow inside the monolith porous zone is treated as laminar due to the very small hydraulic diameter of the flow channels.

The software solves the Reynolds Averaged Navier-Stokes (RANS) equations utilizing the k- $\omega$ turbulence model where $\mathrm{k}$ is the turbulent kinetic energy and $\omega$ is the specific dissipation rate along with the continuity and energy equations for a discretized domain. The governing equations for the flow within the domain are shown below.

The continuity equation is:

$$
\frac{\partial \rho}{\partial t}+\nabla \cdot(\rho v)=0
$$

where $\rho, t$ and $v$ are the density, time and fluid velocity respectively. For the porous zone, the velocity is replaced with the superficial velocity which is equal to the fluid physical velocity inside the porous zone multiplied by the medium porosity.

The Reynolds Average Navier Stokes (RANS) equation can be written as:

$$
\frac{\partial \rho v}{\partial t}+\nabla \cdot(\rho v v)=-\nabla \mathrm{p}+\nabla \cdot \tau-\nabla \cdot\left(\rho \overline{v^{\prime} v^{\prime}}\right)
$$

where $\mathrm{p}, \tau$ and $v^{\prime}$ are the pressure, stress tensor and fluctuating component of the velocity, respectively. The final term on the right-hand side of equation (2) represents the Reynolds stresses term. For the porous zone, the velocity in equation (2) is be replaced with the superficial velocity in addition to changing the Reynold stresses term with $v_{S} * \mu / K$ where $v_{s}$, $\mu$ and $K$ are the superficial velocity, fluid dynamic viscosity and the permeability tensor, respectively. To insure that the flow is unidirectional inside the monolith, the permeability in the traverse (radial) direction is imposed three orders of magnitude lower than the flow direction permeability [14]. According to the Boussinesq assumption, the turbulence stresses can be written as:

$$
-\rho \overline{v^{\prime} v^{\prime}}=\mu_{t}\left(\nabla v+\nabla v^{T}\right)-\frac{2}{3} * \mathrm{k} * \mathrm{I}
$$

where $\mu_{t}, v^{T}, \mathrm{I}$ are the eddy viscosity, fluctuating velocity vector and flow inertia, respectively. The turbulence transport equations are:

$$
\begin{gathered}
\frac{\partial(\rho k)}{\partial t}+\nabla \cdot(\rho k \mathrm{v})=\nabla\left(\theta_{k} \nabla k\right)+G_{k}-Y_{k}+S_{k} \\
\frac{\partial(\rho \omega)}{\partial t}+\nabla \cdot(\rho \omega \mathrm{v})=\nabla\left(\theta_{\omega} \nabla \omega\right)+G_{\omega}-Y_{\omega}+S_{\omega}
\end{gathered}
$$

where $\theta, G, Y$ and $S$ are the effective diffusivity, generation terms, dissipation terms and source terms, respectively. The subscripts $k$ and $\omega$ represent turbulent kinetic energy and specific dissipation rate, respectively. Two separate conservation of energy equations should be defined for both the fluid flow through the monolith and the solid monolith. For the fluid, radiation is neglected and the equation can be written as:

$$
\frac{\partial}{\partial z}\left(k_{e f f}^{f} \frac{\partial}{\partial z} T_{f}\right)-\rho C_{p} v_{s} \frac{\partial T_{f}}{\partial z}=\mathrm{h} A_{v}\left(T_{f}-T_{s}\right)
$$


$k_{e f f}^{f}$ is the effective thermal conductivity of the fluid, $C_{p}$ is the fluid specific heat and $h$ is the convective heat transfer coefficient. $T_{f}$ and $T_{s}$ are the fluid and solid temperatures, respectively. The conduction term in equation 6 (first term in the right-hand side) can be replaced by $\nabla .\left(k_{e f f}^{S} \nabla T_{s}\right)$ where $k_{\text {eff }}^{S}$ is the effective thermal conductivity of the monolith and it varies for both the axial and radial directions. The effective thermal conductivity is calculated using the methodology outlined in [17] and the convective heat transfer coefficient is calculated from equating the Nusselt number equation to 4 as suggested by [14]. The surface to surface radiation is neglected due to the lack of extremely large temperature gradients in the axial direction.

A mass flow inlet condition at prescribed values of mass-flow-rate and temperature is specified and the walls are assigned convection boundary conditions to the ambient with a surrounding temperature of $23^{\circ} \mathrm{C}$ and convective heat transfer coefficient of $21 \mathrm{~W} / \mathrm{m}^{2} \mathrm{~K}$. The outlet is assigned a pressure outlet boundary conditions of $1 \mathrm{~atm}$.

\section{Results and discussion}

The fluid flow domain is discretized using ANSYS' built in meshing algorithms. An initial grid independence study was performed on a 3-Dimensional model of the catalytic converter using three different meshes with 150,000 nodes, 800,000 nodes and 2.5 million nodes. The study demonstrated that a mesh larger than 800,000 nodes only shows an RMSD (Root Mean Square Deviation) of $\pm 1.4 \%$ in the velocity predictions and $\pm 0.6 \%$ for the temperature predictions behind the substrate. In order to minimize computational time, a two-dimensional axisymmetric case was compared to the 3 -dimensional simulations. The two-dimensional mesh had a total of 210,000 nodes and was constructed using similar element sizing as the 800,000 3-D mesh. The 2-D mesh showed a deviation (RMSD) of $\pm 7.5 \%$ for temperature predictions and $\pm 6 \%$ for velocity predictions from the 800,0003 -D mesh. The difference in the predicted velocity and temperature profiles behind the monolith that were used for the grid independence study is shown in Figure 2. The 2D mesh was utilised for the remainder of this study.

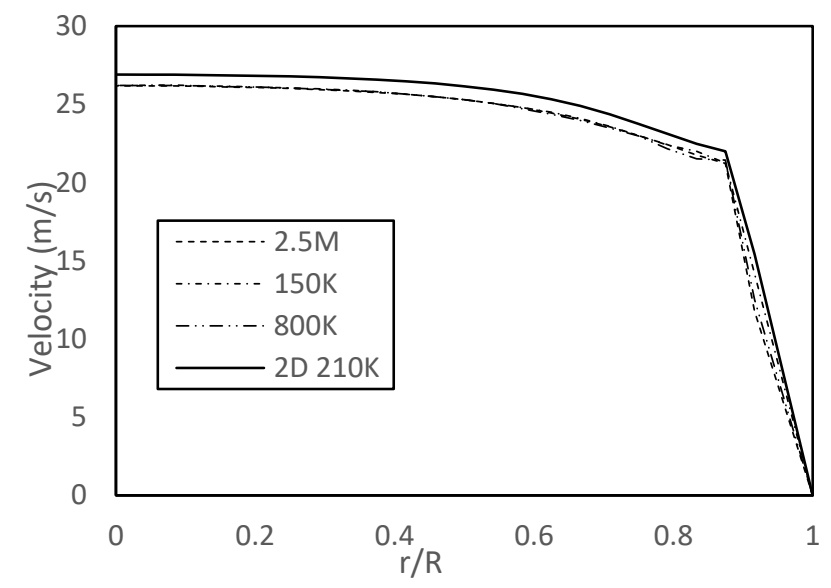

(a)

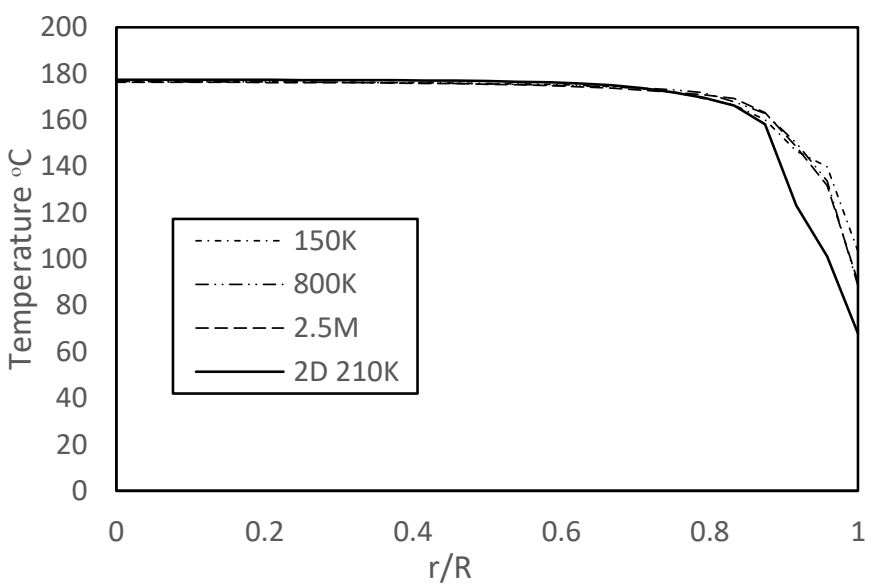

(b)

Fig. 2: Grid independence study at mass flow rate of $0.072 \mathrm{~kg} / \mathrm{s}$ and inlet temperature of $177^{\circ}$ (a) velocity analysis and (b) temperature analysis.

The flow and temperature fields are shown in Figure 3 where the flow direction is from left to right and the grey areas in (a), (b), (d) and (e) represent the solid insulation padding. The velocity streamlines show the behaviour of the flow in the inlet diffuser and outlet cone. Differences in pressure near the diffuser walls cause flow circulation in the expansion region of the diffuser (see Figure $3 \mathrm{~b}$ and c). The region behind the insulation padding shows very low velocities as the flow is blocked from the upstream causing a shear layer and flow circulation to develop. The velocity vectors (see Figure $3 \mathrm{c}$ ) show the effect of the monolith presence on the flow velocity distribution at the inlet face. The monolith acts as a flow resistance zone creating a high pressure area in the centre of the catalytic converter (Figure 3e) and forcing flow redistribution to the sides. 


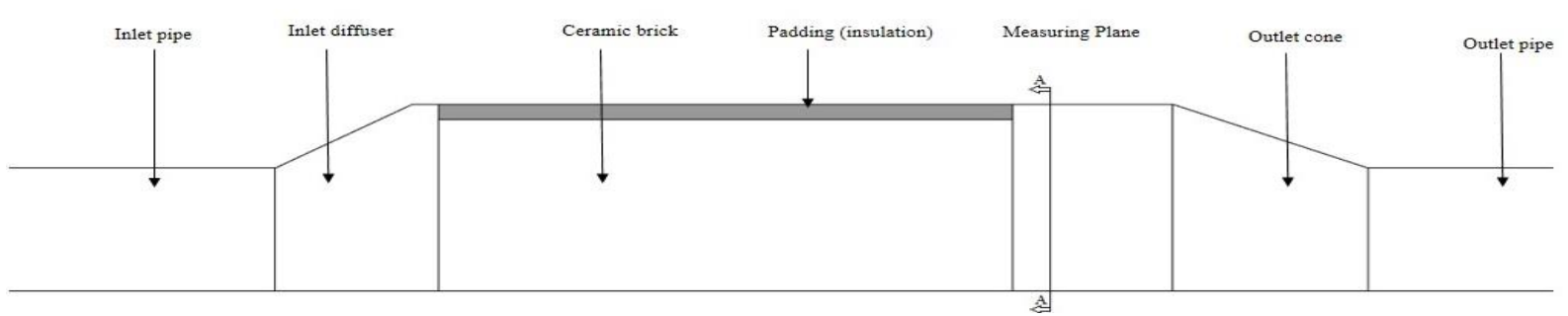

(a)

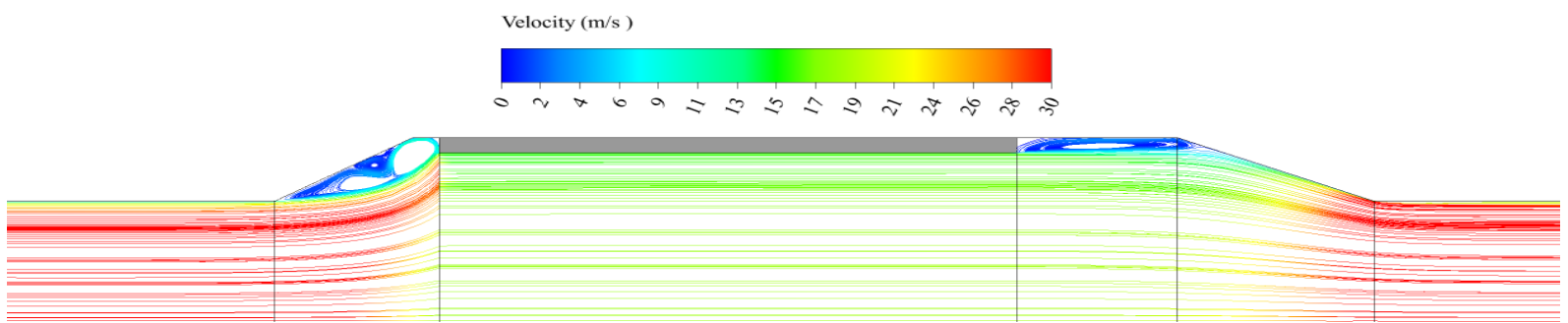

(b)

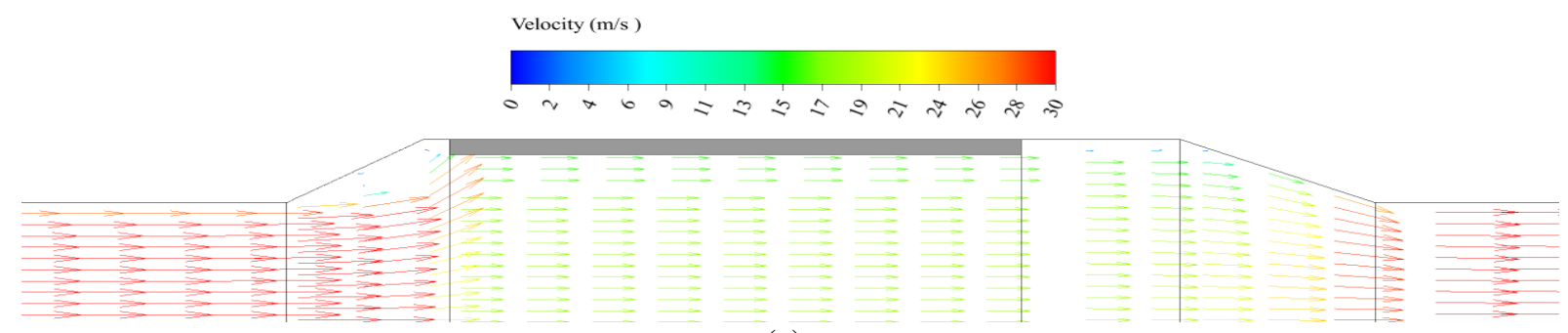

(c)

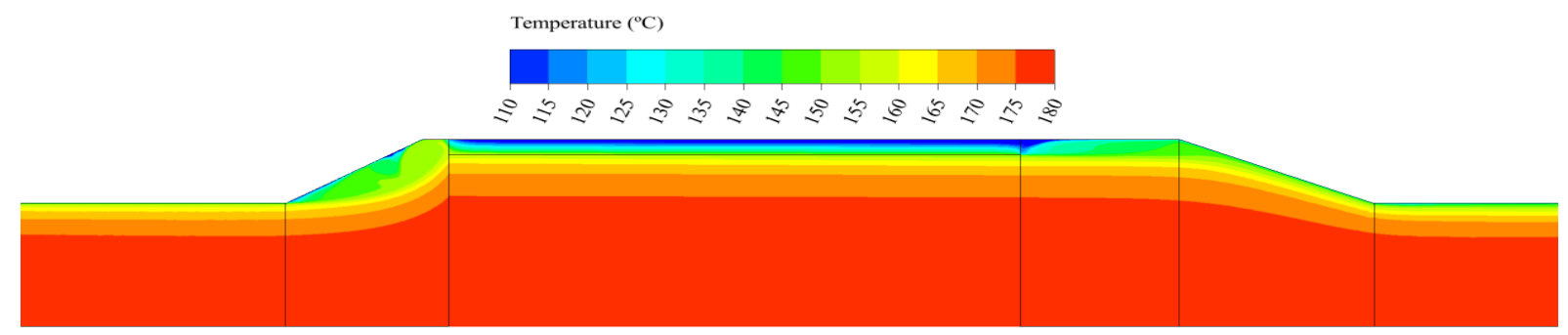

(d)

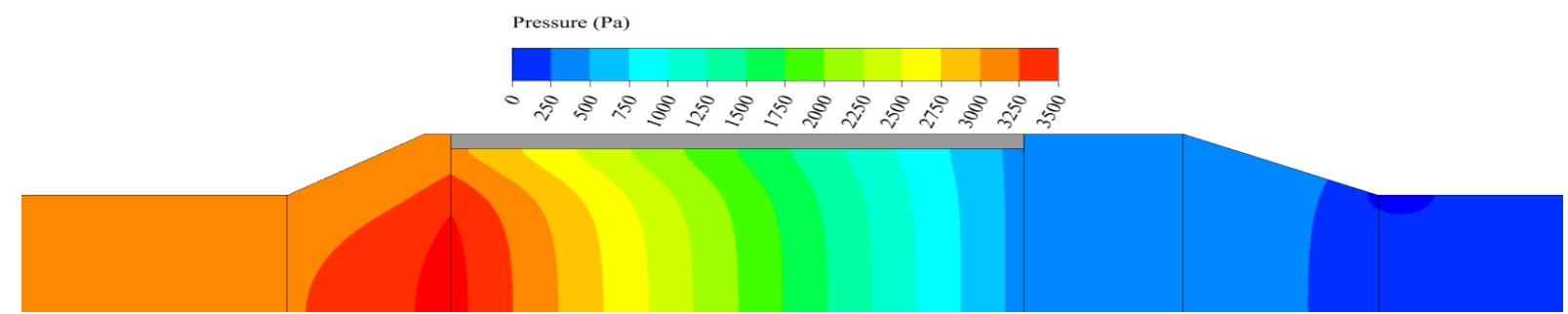

(e)

Fig. 3: CFD simulation of air flow through a model catalytic converter at mass flow rate of $0.05 \mathrm{~kg} / \mathrm{s}$ and inlet temperature of $177^{\circ} \mathrm{C}$, (a) computational domain schematic, (b) velocity streamline, (c) velocity vectors, (d) temperature contours and (e) pressure contours.

Addition of the thermal energy component into the catalytic converter flow simulation gives a better understanding of the flow field as temperature affects fluid properties such as the density and viscosity. Observed temperature gradients 
across the width of the catalytic converter (Figure 3d) reflects on the observed velocity gradient (Figure 3c). Regions with low velocities due to high vorticity and flow circulation tend to have lower temperature, while high velocity regions typically exhibit high temperatures. Overall the monolith shows high temperature uniformity, with a drop-off close to the walls due to heat loss to external convection and the insulation padding. This is expected for steady state condition as the convection losses are relatively low.

The simulations are validated against experimental local temperature and velocity measurements. The validation is shown in Figure 4 below.

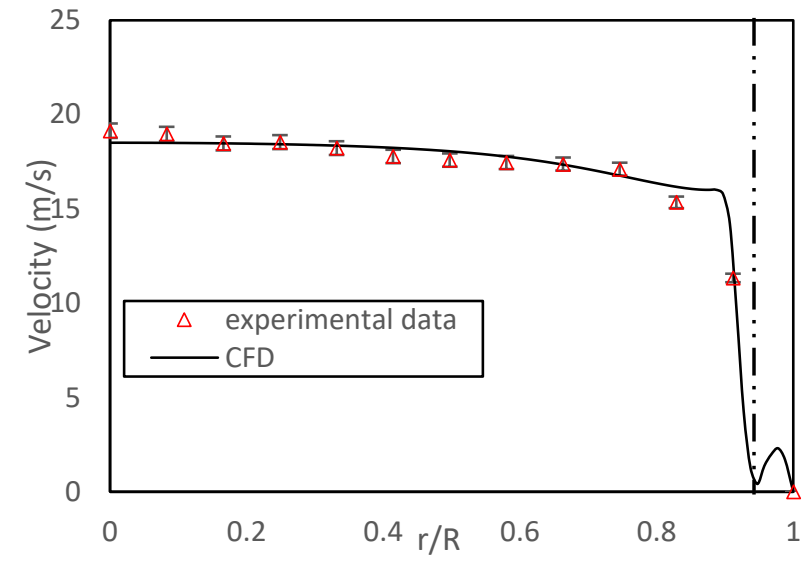

(a)

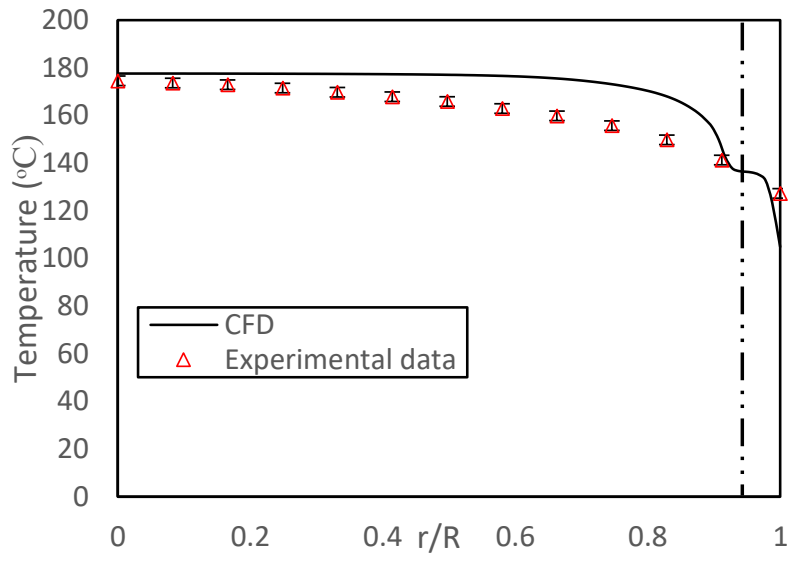

(b)

Fig. 4: Comparison of experimental measurements (symbols) and CFD simulation (line) of. (a) velocity and (b) temperature across the width of the model catalytic converter system. Error bars represent the standard deviation of 4 repeated measurements. Measurement is conducted $0.5 \mathrm{~cm}$ behind the monolith. Inlet flow was $0.05 \mathrm{~kg} / \mathrm{s}$ and inlet temperature of $177^{\circ} \mathrm{C}$. The dashed vertical line represents the boundary between the monolith and the padding. $r$ represents the measurement location (radius), while $\mathrm{R}$ is the inner radius of the catalytic converter.

Computed velocities are in good agreement with the experimental data (RMSE of $\pm 5 \%$ ) and show no apparent bias. Computed temperatures show a positive bias over the measured temperature profile, even though the overall agreement is good (RMSE of $\pm 8 \%$ ). The temperature model is under further investigation to attempt to rectify this bias.

\section{Conclusion}

Accurate prediction of flow velocity and temperature fields is critical for the simulation of catalytic converter emissions performance as these are the key input for a chemical kinetics model.

The work presented in this study successfully simulates the flow through a catalytic converter with good agreement with experimental measurements. Positive temperature bias will be investigated further. Utilizing the k- $\omega$ turbulence model showed robust convergence and great stability in the CFD modeling. Further development of this model will include study of transient conditions and incorporation of a chemical reaction to provide a comprehensive tool to evaluate the emissions performance of automotive catalytic converters.

\section{Acknowledgements}

The authors wish to acknowledge the support from the Natural Sciences and Engineering Research Council of Canada (NSERC), Mitacs and Ontario Center of Excellence (OCE) during the course of this study. The support of Vida Fresh Air Corp. is also highly appreciated.

\section{References}

[1] M. V. Twigg, "Progress and future challenges in controlling automotive exhaust gas emissions," Appl. Catal. B Environ., vol. 70, no. 1-4, pp. 2-15, 2007.

[2] V. Tomašić, "Application of the monoliths in DeNOx catalysis," Catal. Today, vol. 119, no. 1-4, pp. 106-113, 2007. 
[3] B. Cho, "Dynamic Behavior of a Single Catalyst Pellet. 1. Symmetric Concentration Cycling during CO Oxidation over Pt/AI2O3," no. 1980, pp. 410-420, 1983.

[4] R. M. Heck, S. Gulati, and R. J. Farrauto, "The application of monoliths for gas phase catalytic reactions," Chem. Eng. J., vol. 82, no. 1-3, pp. 149-156, 2001.

[5] M. Bella, G. Rocco, V. Maggiore, "A study of inlet flow distortion effects on automotive catalytic converters," $J$. Eng. Gas Turbines Power, 1991.

[6] D. Lai, M. Kim, Y. Cheng, C. Li, P. Chui, G. Pakko, "Three dimensional simulations of automotive catalytic conveter internal flow.pdf," SAE Tech. Pap., pp. 1-10, 1991.

[7] M. C. Lai, T. Lee, J. Y. Kim, C. Y. Cheng, P. Li, and G. Chui, "Numerical and experimental characterizations of automotive catalytic converter internal flows," J. Fluids Struct., vol. 6, no. 4, pp. 451-470, 1992.

[8] S. H. Amirnordin, S. M. Seri, W. S. W. Salim, H. A. Rahman, and K. Hasnan, "Pressure drop analysis of square and hexagonal cells and its effects on the performance of catalytic converters," Int. J. Environ. Sci. Dev., vol. 2, no. 3, pp. 239-247, 2011.

[9] E. Karvounis and D. N. Assanis, "The effect of inlet flow distribution on catalytic conversion efficiency," Int. J. Heat Mass Transf., vol. 36, no. 6, pp. 1495-1504, 1993.

[10] D. Weltens, H. Bressler, H. Terres, F. Neumaier, H. Rammoser, "Optimisation of Catalytic Converter Gas Flow Distribution by CFD Prediction," SAE Tech. Pap., 1993.

[11] K. Ramanathan, V. Balakotaiah, and D. H. West, "Light-off criterion and transient analysis of catalytic monoliths," Chem. Eng. Sci., vol. 58, no. 8, pp. 1381-1405, 2003.

[12] S. Jeong and W. Kim, "A Numerical Approach to Investigate Transient Thermal and Conversion Characteristics of Automotive Catalytic Converter," no. 724, 1998.

[13] A. P. Martin, N. S. Will, A. Bordet, P. Cornet, C. Gondoin, and X. Mouton, "Effect of flow distribution on emissions performance of catalytic converters," SAE Tech. Pap., vol. 980936, no. 724, pp. 980936, 1998.

[14] R. E. Hayes, A. Fadic, J. Mmbaga, and A. Najafi, "CFD modelling of the automotive catalytic converter," Catal. Today, vol. 188, no. 1, pp. 94-105, 2012.

[15] K. Chen, Ming; Schirmer, “A Modelling Approach To the Design Optimization of Catalytic,” pp. 1-7, 2003.

[16] W. Guojiang and T. Song, "CFD simulation of the effect of upstream flow distribution on the light-off performance of a catalytic converter," Energy Convers. Manag., vol. 46, no. 13-14, pp. 2010-2031, 2005.

[17] C. G. Visconti, G. Groppi, and E. Tronconi, "Accurate prediction of the effective radial conductivity of highly conductive honeycomb monoliths with square channels," Chem. Eng. J., vol. 223, pp. 224-230, 2013. 